\title{
Severe C8 or T1 Symptoms after Cervical Laminoplasty and Related Factors: Are There Any Differences between C3-C6 Laminoplasty and C3-C7 Laminoplasty?
}

\author{
Hitoshi Kudo ${ }^{1}$, Kazunari Takeuchi ${ }^{2}$, Toru Yokoyama ${ }^{2}$, Yoshihito Yamasaki ${ }^{3}$, Kanichiro Wada ${ }^{1}$, \\ Gentaro Kumagai ${ }^{1}$, Toru Asari ${ }^{1}$, Hironori Otsuka ${ }^{4}$, Yasuyuki Ishibashi ${ }^{1}$ \\ ${ }^{1}$ Department of Orthopaedic Surgery, Hirosaki University Graduate School of Medicine, Hirosaki, Japan \\ ${ }^{2}$ Department of Orthopaedic Surgery, Odate Municipal General Hospital, Odate, Japan \\ ${ }^{3}$ Department of Orthopaedic Surgery, Aomori City Hospital, Aomori, Japan \\ ${ }^{4}$ Department of Orthopaedic Surgery, Japan Community Health Care Organization Akita Hospital, Noshiro, Japan
}

\begin{abstract}
Study Design: Retrospective study.
Purpose: We experienced the situation wherein some patients had new-onset pain or dysesthesia around the ring and little fingers (C8 symptom) or ulnar aspect of the forearm (T1 symptom) after cervical laminoplasty (LP). We investigated the incidence and the cause of new C8 or T1 symptoms and the clinical outcomes after C3-C6 LP or C3-C7 LP.

Overview of Literature: There were some reports regarding complications after cervical LP. However, there was no report regarding C8 or T1 symptoms after cervical LP.

Methods: Among the 33 patients enrolled in this study, 11 and 22 patients were treated with C3-C6 LP and C3-C7 LP, respectively. We prospectively evaluated C8 or T1 symptoms daily postoperatively for 1 week. The distance of the posterior spinal cord shifting and posterior subarachnoid space from C2 to T1 was measured by T2-weighted midsagittal magnetic resonance imaging (MRI). We evaluated pre- and postoperative axial neck pain, Japanese Orthopaedic Association (JOA) score, and JOA score improvement rate.

Results: C8 or T1 symptoms occurred in five and three patients with C3-C6 LP (45.5\%) and C3-C7 LP (13.6\%), respectively. The distance of the posterior subarachnoid space in C3-C6 LP at C7 was significantly shorter than that in C3-C7 LP at T1 on MRI 24 hours postoperatively $(p=0.0448)$. Postoperative axial neck pain, pre- and postoperative JOA scores, and JOA score improvement rate were not significantly different.

Conclusions: The incidence of C8 or T1 symptoms in C3-C6 LP was higher than that in C3-C7 LP. C8 or T1 symptoms would be caused by the posterior fila radicularia and spinal cord impingement on the intact lower end of the lamina.
\end{abstract}

Keywords: C8 symptom; T1 symptom; Cervical spondylotic myelopathy; Cervical laminoplasty

Received Sep 25, 2018; Revised Nov 28, 2018; Accepted Dec 17, 2018

Corresponding author: Hitoshi Kudo

Department of Orthopaedic Surgery, Hirosaki University Graduate School of Medicine, 5 Zaifu-cho, Hirosaki 036-8562, Japan

Tel: +81-172-39-5083, Fax: +81-172-36-3826, E-mail: hitoshikudo@hirosaki-u.ac.jp 


\section{Introduction}

Cervical laminoplasty (LP) has been reported to produce stable long-term neurological improvement for cervical myelopathy [1]. In the department of orthopaedic surgery of Hirosaki University Graduate School of Medicine and related institutions, C3-C7 double-door LP has also been adapted to cervical myelopathy since 1986, when we introduced LP as treatment for cervical myelopathy [2]. However, several postoperative complications, including C5 palsy [3], postoperative axial neck pain, and limitation of activity of daily life due to reduced neck mobility, have been reported [4]. Particularly, many studies have reported on the causes and prophylaxes of postoperative axial neck pain, and nuchal muscles have been preserved. Selective cervical LP has been reported to preserve nuchal muscles as much as possible, remarkably reducing postoperative axial neck pain [5]. The preservation of the semispinalis cervicis (SSC) in $\mathrm{C} 2$ has been reported to effectively reduce postoperative axial neck pain after LP [6]. In contrast, an anatomical study has reported that many nuchal ligaments were attached to the $\mathrm{C} 7$ spinous process, and the preservation of the $\mathrm{C} 7$ spinous process in LP was recommended to reduce postoperative axial neck pain [7]. Furthermore, several clinical studies have reported that postoperative axial neck pain after C3-C6 LP was rarer than that after conventional C3-C7 LP [8]. Based on these reports, we agreed to perform C3-C6 LP to preserve the nuchal ligaments attached to the C7 spinous process, and we adopted C3-C6 LP for the cases that satisfied our criteria from 2006 [9].

The diameter of the dural sac at C6/7 as shown on postoperative magnetic resonance imaging (MRI) in C3-C6 LP was reported to be significantly smaller than that in C3-C7 LP [7]. In addition, we experienced the situation wherein the diameter of the dural sac at $\mathrm{C} 6 / 7$ in $\mathrm{C} 3-\mathrm{C} 6 \mathrm{LP}$ was significantly smaller than that in C3-C7 LP on MRI postoperatively. Moreover, after C3-C6 LP, some patients complained of new-onset pain or dysesthesia around the ring and little fingers (C8 area) or the ulnar aspect of the forearm (T1 area), namely C8 or T1 symptoms. We hypothesized that the incidence of $\mathrm{C} 8$ or $\mathrm{T} 1$ symptoms in C3-C6 LP was higher than that in C3-C7 LP.

This study aimed to investigate the incidence of new $\mathrm{C} 8$ or T1 symptoms after LP, comparing quantitatively the distance of the spinal cord posterior shifting and posterior subarachnoid space between C3-C6 LP and C3-C7 LP on MRI postoperatively [10], and to investigate the cause of
C8 or T1 symptoms. In addition, we aimed to investigate the differences in the Visual Analog Scale (VAS) score of axial neck pain, Japanese Orthopaedic Association (JOA) score pre- and postoperatively, and JOA score improvement rate between the two groups.

\section{Materials and Methods}

\section{Patient population}

The institutional review board of the Japan Community Health Care Organization Akita Hospital approved the study (IRB approval no., 1026009), and written informed consents were obtained.

This study enrolled 33 consecutive patients between August 2006 and January 2011. Eleven patients (8 men; 3 women; mean age, 61 years; range, $42-84$ years) with cervical spondylotic myelopathy (CSM) were treated with C4-C6 LP with C3 laminectomy (LN), preserving SSC and $\mathrm{C} 7$ spinous process, and were designated as the $\mathrm{C} 3-$ C6 group. In addition, 22 patients ( 13 men; 9 women; mean age, 66 years; range, 34-81 years) with CSM were treated with $\mathrm{C} 4-\mathrm{C} 7 \mathrm{LP}$ with $\mathrm{C} 3 \mathrm{LN}$ and were designated as the $\mathrm{C} 3-\mathrm{C} 7$ group.

Our criteria for C3-C6 LP were (1) the absence of myelopathy at $\mathrm{C} 6-\mathrm{C} 7,(2)>1 \mathrm{~mm}$ posterior subarachnoid space at C6-C7 on MRI preoperatively, and (3) no ossification of the posterior longitudinal ligament (OPLL) [9]. Thus, we excluded patients with OPLL who underwent C3-C7 LP. The patients who underwent cervical anterior fusion and any other cervical spine surgery were also excluded.

\section{Operative technique and postoperative management}

In all cases of both of the groups, LN was performed at $\mathrm{C} 3$, and SSC muscle insertion in C2 was completely preserved [6]. The laminoplastic procedure was performed at $\mathrm{C} 4-\mathrm{C} 6$ and $\mathrm{C} 4-\mathrm{C} 7$ in the $\mathrm{C} 3-\mathrm{C} 6$ and $\mathrm{C} 3-\mathrm{C} 7$ groups. In the $\mathrm{C} 3-\mathrm{C} 6$ group, $\mathrm{C} 7$ spinous process was preserved. In the $\mathrm{C} 3-\mathrm{C} 7$ group, the nuchal ligament attachment site of the $\mathrm{C} 7$ spinous process was preserved, cut, and separated the top of the $\mathrm{C} 7$ spinous process off the $\mathrm{C} 7$ lamina, and adopted the $\mathrm{C} 7$ laminoplastic procedure. The laminoplastic procedure was adopted from the spinous process-splitting (double-door) LP by using hydroxyapatite spinous process spacers [2], and the spinous process was split using a thread-wire saw. We excised the cephalic 

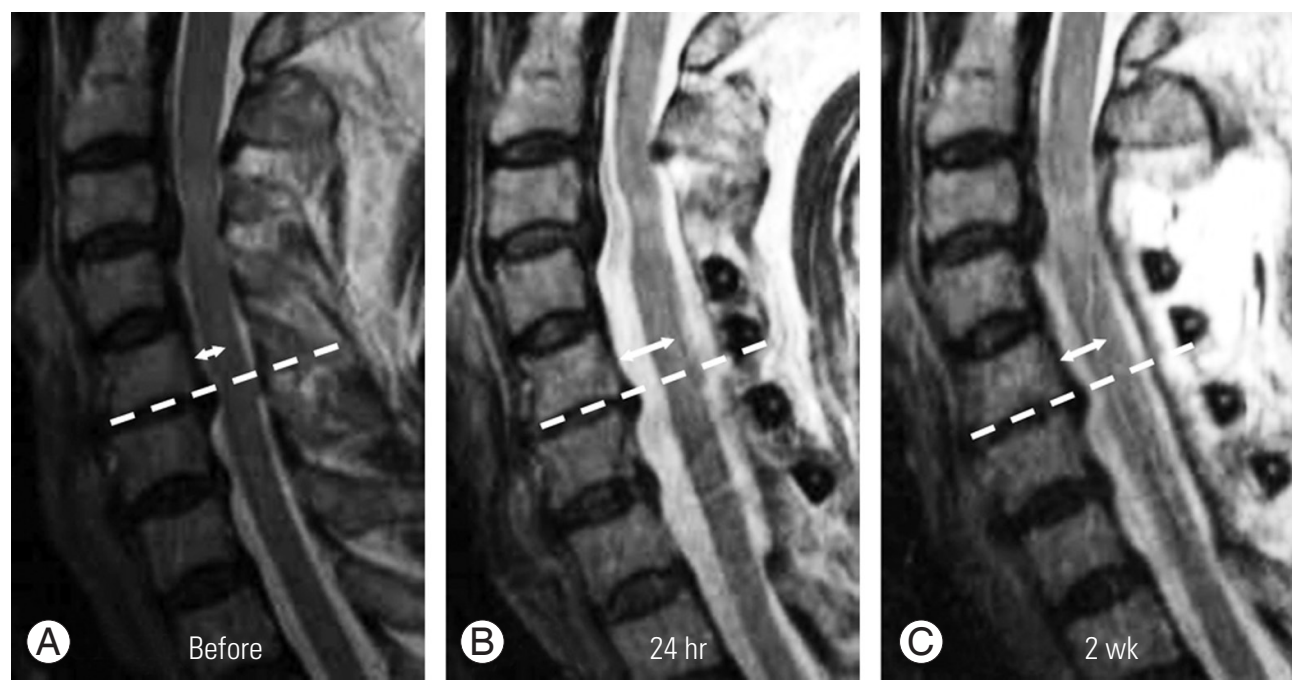

Fig. 1. Distance of the posterior spinal cord shifting. (A-C) The measurement of the distance from the posterior edge of each vertebral body to the center of the spinal cord. Distance of the posterior spinal cord shifting=distance at 24 hours or 2 weeks postoperatively-preoperative distance.

end of C7 or T1 lamina to pass the thread-wire saw guide, not to decompression. We sutured the $\mathrm{C} 7$ bone fragments attached to the nuchal ligaments by using non-absorbable suture in the $\mathrm{C} 3-\mathrm{C} 7$ group.

We did not adapt the postoperative neck collar to any patients. The suction tube drain for postoperative bleeding was put between the lamina and deep muscle, and it was removed within 2 days postoperatively. Exercise was started 2 days postoperatively.

We defined new-onset pain or dysesthesia postoperatively around the ring and little fingers and the ulnar aspect of the forearm as $\mathrm{C} 8$ and $\mathrm{T} 1$ symptoms, respectively. In all the patients, postoperative $\mathrm{C} 5$ palsy, C8 symptom, and $\mathrm{T} 1$ symptom were evaluated daily postoperatively for 1 week.

\section{Measurement of the spinal cord movement pre- and postoperatively with magnetic resonance imaging}

All the patients were prospectively examined with MRI (EXELART, 1.5T; Toshiba Co. Ltd., Tokyo, Japan) preoperatively, and 24 hours, and 2 weeks postoperatively [10]. ImageJ (http://rsbweb.nih.gov/ij/) was used to measure the following three items in all the T2-weighted midsagittal MRI. The distance from the posterior edge of each vertebral body to the center of the spinal cord (Fig. 1) and the distance of the posterior subarachnoid space (Fig. 2) were measured from $\mathrm{C} 2$ to $\mathrm{T} 1$. The following equation was used to determine the distance of posterior spinal cord shifting: posterior spinal cord shifting $(\mathrm{mm})=$ distance

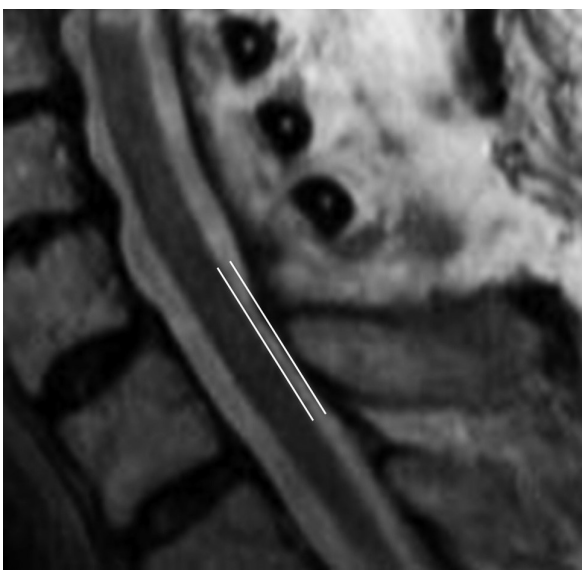

Fig. 2. Distance of the posterior subarachnoid space. The measurement of the distance of posterior subarachnoid space from the posterior edge of the spinal cord to the posterior edge of the dura mater.

from the posterior edge of each vertebral body to the center of the spinal cord at 24 hours and 2 weeks postoperatively-distance preoperatively.

\section{Evaluation of pre- and postoperative axial neck pain and Japanese Orthopaedic Association score}

We used VAS (0-100 $\mathrm{mm})$ to evaluate pre- and postoperative axial neck pain. In addition, we evaluated the preand postoperative JOA scores. Postoperative axial neck pain and JOA score were evaluated 1 year postoperatively, and the JOA score improvement rate was calculated using the Hirabayashi method. 
Table 1. The patients with C8 or T1 symptoms

\begin{tabular}{|c|c|c|c|c|c|}
\hline Case & Age (yr) & $\operatorname{Sex}(M / F)$ & Surgical procedure & Symptom & Side \\
\hline 1 & 42 & M & C3 LN+C4-6 LP & $\mathrm{T} 1$ & Left \\
\hline 2 & 47 & M & C3 LN+C4-6 LP & C8 & Left \\
\hline 3 & 75 & $\mathrm{~F}$ & C3 LN+C4-6 LP & C8 & Left \\
\hline 4 & 61 & M & C3 LN+C4-6 LP & $\mathrm{T} 1$ & Right \\
\hline 5 & 72 & M & C3 LN+C4-6 LP & C8 & Left \\
\hline 6 & 49 & $\mathrm{~F}$ & C3 LN+C4-7 LP & C8 & Right \\
\hline 7 & 70 & $\mathrm{~F}$ & C3 LN+C4-7 LP & C8 & Left \\
\hline 8 & 73 & M & C3 LN+C4-7 LP & $\mathrm{T} 1$ & Right \\
\hline
\end{tabular}

M, male; F, female; LN, laminectomy; LP, laminoplasty.

Table 2. The distance of the posterior spinal cord shifting

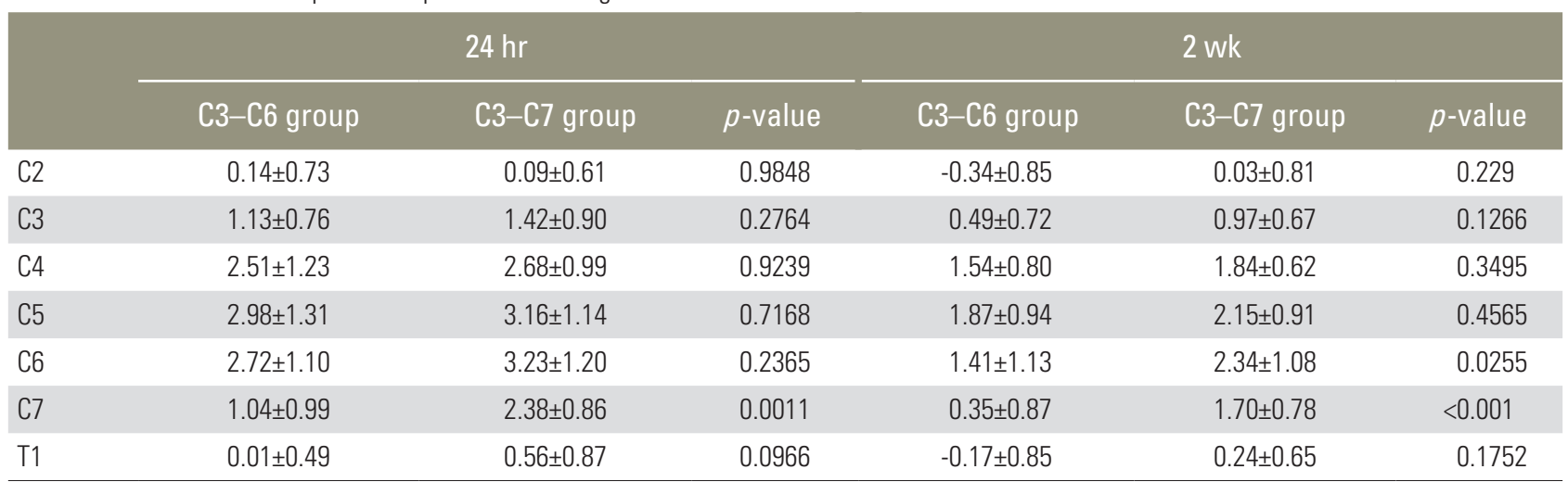

Values are presented as mean \pm standard deviation $(\mathrm{mm})$. All $p$-values were calculated by the Mann-Whitney U-test.

\section{Statistical analysis}

Chi-square test was used to analyze the incidence of $\mathrm{C} 8$ or $\mathrm{T} 1$ symptoms between the $\mathrm{C} 3-\mathrm{C} 6$ and $\mathrm{C} 3-\mathrm{C} 7$ groups. The Mann-Whitney $U$-test was used to analyze the distance of the spinal cord posterior shifting and posterior subarachnoid space, VAS for axial neck pain, JOA score, and JOA score improvement rate. All $p<0.05$ were considered statistically significant.

\section{Results}

\section{Incidence of C8 or T1 symptoms}

C8 or T1 symptoms occurred in five patients in the C3-C6 group (45.5\%), and in three patients in the $\mathrm{C} 3-\mathrm{C} 7$ group (13.6\%) within 1 week postoperatively. C8 or T1 symptoms in the C3-C6 group were significantly more frequent than those in the $\mathrm{C} 3-\mathrm{C} 7$ group $(p=0.04437)$. Only one side was affected in all cases of both of the groups. Almost all C8 or T1 symptoms were very mild and disappeared within 2-3 weeks. Only one patient in the C3-C6 group experienced $\mathrm{T} 1$ symptom with severe pain and functional motility disorder of the ipsilateral lower extremity, which was suspected as a long tract sign. C5 palsy occurred in one patient in the $\mathrm{C} 3-\mathrm{C} 7$ group (Table 1).

\section{Distance of the posterior spinal cord shifting}

Table 2 shows the distance of the posterior spinal cord shifting at 24 hours and 2 weeks postoperatively. A significant difference was found at C7 ( $p=0.0011)$; however, no significant difference was found at C6 ( $p=0.2365)$ at 24 hours postoperatively. Moreover, significant differences were found at C6 $(p=0.0255)$ and C7 $(p<0.001)$ at 2 weeks postoperatively. These data suggested that the distance of the posterior spinal cord shifting at C6 in the C3-C6 group is similar to that in the $\mathrm{C} 3-\mathrm{C} 7$ group in the early postoperative period. 
Table 3. The distance of the posterior subarachnoid space

\begin{tabular}{|c|c|c|c|c|c|c|c|c|c|}
\hline & \multicolumn{3}{|c|}{ Before } & \multicolumn{3}{|c|}{$24 \mathrm{hr}$} & \multicolumn{3}{|c|}{2 wk } \\
\hline & C3-C6 group & C3-C7 group & $p$-value & C3-C6 group & C3-C7 group & $p$-value & C3-C6 group & C3-C7 group & $p$-value \\
\hline $\mathrm{C} 2$ & $1.91 \pm 0.58$ & $1.80 \pm 0.72$ & 0.4563 & $1.52 \pm 0.64$ & $1.36 \pm 0.64$ & 0.5407 & $1.69 \pm 0.83$ & $1.36 \pm 0.60$ & 0.2591 \\
\hline C3 & $1.48 \pm 0.38$ & $1.53 \pm 0.55$ & 0.8935 & $2.65 \pm 0.74$ & $2.44 \pm 0.68$ & 0.5286 & $2.24 \pm 0.38$ & $2.30 \pm 0.70$ & 0.6743 \\
\hline C4 & $1.20 \pm 0.44$ & $1.33 \pm 0.55$ & 0.3890 & $3.23 \pm 1.07$ & $3.13 \pm 0.81$ & 1.000 & $3.13 \pm 0.77$ & $3.06 \pm 0.84$ & 0.7310 \\
\hline C5 & $1.02 \pm 0.45$ & $1.46 \pm 0.74$ & 0.0927 & $3.28 \pm 1.01$ & $3.30 \pm 0.97$ & 1.000 & $2.82 \pm 0.58$ & $2.90 \pm 0.94$ & 0.8187 \\
\hline C6 & $0.97 \pm 0.55$ & $1.00 \pm 0.47$ & 0.8038 & $3.50 \pm 0.70$ & $3.42 \pm 1.05$ & 0.8936 & $3.09 \pm 0.60$ & $2.86 \pm 0.74$ & 0.3395 \\
\hline $\mathrm{C7}$ & $1.85 \pm 0.55$ & $1.20 \pm 0.45$ & 0.0033 & $0.77 \pm 0.77$ & $3.29 \pm 1.01$ & $<0.001$ & $1.34 \pm 0.45$ & $2.67 \pm 0.66$ & $<0.001$ \\
\hline T1 & $2.09 \pm 0.75$ & $1.89 \pm 0.62$ & 0.9543 & $2.25 \pm 0.74$ & $1.27 \pm 0.45$ & 0.0469 & $2.12 \pm 0.77$ & $1.52 \pm 0.50$ & 0.2674 \\
\hline
\end{tabular}

Values are presented as mean \pm standard deviation $(\mathrm{mm})$. All $p$-values were calculated by the Mann-Whitney U-test.

Table 4. The distance of the posterior subarachnoid space at the intact lower end of lamina

\begin{tabular}{|c|c|c|c|c|c|c|c|c|}
\hline \multicolumn{3}{|c|}{ Before } & \multicolumn{3}{|c|}{$24 \mathrm{hr}$} & \multicolumn{3}{|c|}{$2 \mathrm{wk}$} \\
\hline $\begin{array}{l}\text { C3-C6 group } \\
\text { (C7) }\end{array}$ & $\begin{array}{l}\text { C3-C7 group } \\
\text { (T1) }\end{array}$ & $p$-value & $\begin{array}{l}\text { C3-C6 group } \\
\text { (C7) }\end{array}$ & $\begin{array}{l}\text { C3-C7 group } \\
\text { (T1) }\end{array}$ & $p$-value & $\begin{array}{l}\text { C3-C6 group } \\
\text { (C7) }\end{array}$ & $\begin{array}{l}\text { C3-C7 group } \\
\text { (T1) }\end{array}$ & $p$-value \\
\hline $1.85 \pm 0.55$ & $1.89 \pm 0.62$ & 0.8039 & $0.77 \pm 0.77$ & $1.27 \pm 0.45$ & 0.0448 & $1.34 \pm 0.45$ & $1.52 \pm 0.50$ & 0.2593 \\
\hline
\end{tabular}

Values are presented as mean \pm standard deviation (mm). All $p$-values were calculated by the Mann-Whitney U-test.

\section{Distance of the posterior subarachnoid space}

The distance of the posterior subarachnoid space was significantly different at $\mathrm{C} 7$ preoperatively between both of the groups ( $p=0.0033$ ) due to our criterion for C3-C6 LP. At 24 hours and 2 weeks postoperatively, the distance at $\mathrm{C} 7$ in the $\mathrm{C} 3-\mathrm{C} 6$ group was significantly shorter than that in the C3-C7 group $(p<0.001, p<0.001)$. However, no significant difference was found at C6 between both of the groups ( $p=0.8936, p=0.3395$ ) (Table 3 ).

At the intact lower end of the lamina, the distance of the posterior subarachnoid space at $\mathrm{C} 7$ in the $\mathrm{C} 3-\mathrm{C} 6$ group $(0.77 \pm 0.77 \mathrm{~mm})$ was significantly shorter than that at $\mathrm{T} 1$ in the $\mathrm{C} 3-\mathrm{C} 7$ group $(1.27 \pm 0.45 \mathrm{~mm})$ at 24 hours postoperatively ( $p=0.0448)$; however, no significant difference was observed at 2 weeks postoperatively ( $p=0.2593$ ) (Table 4). These data suggested that the posterior subarachnoid space in the C3-C6 group was smaller than that in the C3-C7 group at the intact lower end of the lamina in the early postoperative period.

Fig. 3 shows the distance of the posterior subarachnoid space at $\mathrm{C} 7$ and $\mathrm{T} 1$ in the $\mathrm{C} 3-\mathrm{C} 6$ and $\mathrm{C} 3-\mathrm{C} 7$ groups at 24 hours postoperatively. Some patients with small distance of posterior subarachnoid space had no C8 or T1 symptoms.

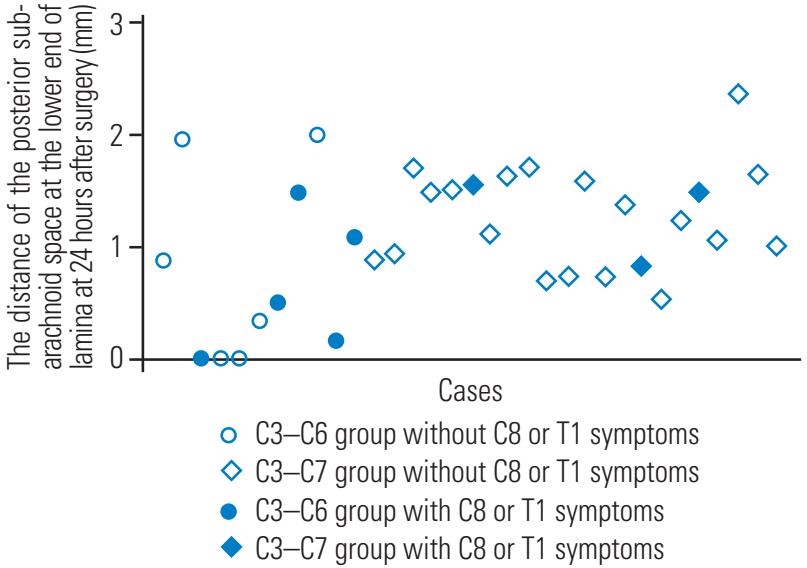

Fig. 3. Distance of the posterior subarachnoid space at the lower end of the lamina in all cases at 24 hours postoperatively. The vertical axis was the distance of the posterior subarachnoid space at the lower end of the lamina at 24 hours postoperatively. The white circle represents the C3-C6 group without C8 or T1 symptoms, and the black circle represents the C3-C6 group with C8 or T1 symptoms. The white rhomboid represents the $\mathrm{C} 3-\mathrm{C} 7$ group without $\mathrm{C} 8$ or $\mathrm{T} 1$ symptoms, and the black rhomboid represents the C3-C7 group with C8 or T1 symptoms.

\section{Evaluation of pre- and postoperative axial neck pain and Japanese Orthopaedic Association score}

Preoperative axial neck pain was observed in eight of 11 patients (72.7\%) and 11 of 22 patients (50.0\%) in the C3- 

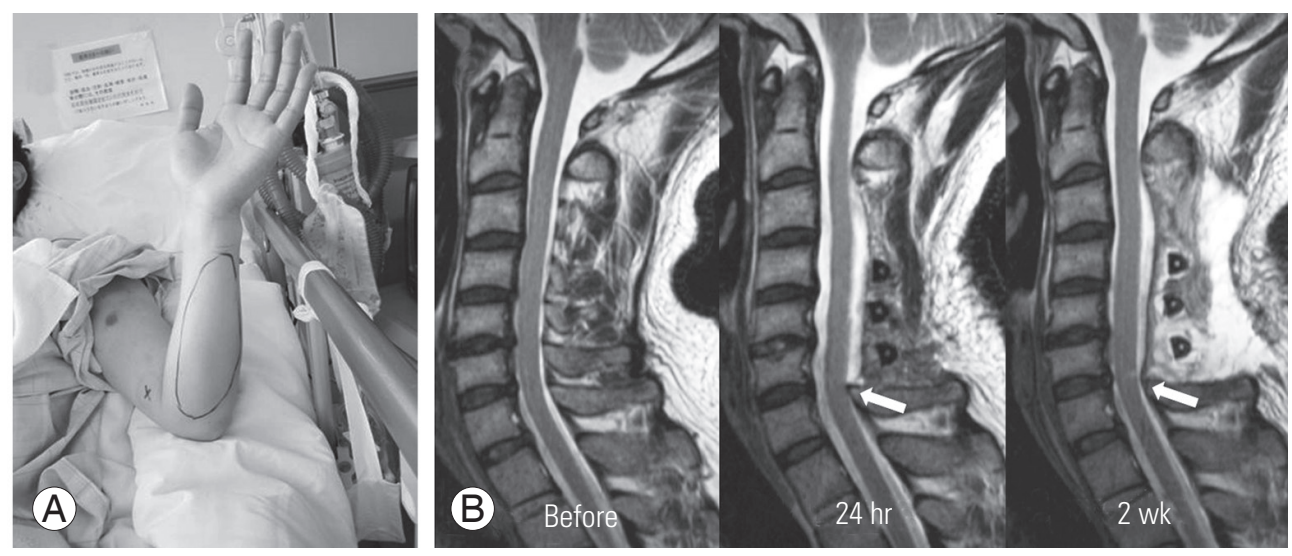

Fig. 4. Case 1 with severe T1 symptom. (A) The patient complained of severe pain around the ulnar aspect of the left forearm (circle, painful area). (B) The spinal cord shifted posteriorly and impinged on the intact C7 lamina on magnetic resonance imaging at 24 hours postoperatively. The spinal cord shifted anteriorly and did not impinge on the lamina 2 weeks postoperatively; however, the high-signal change appeared at C6-C7. Spinal cord or posterior fila radicularia would impinge on the lower end of the lamina (arrow).

C6 and C3-C7 groups, respectively. The mean VAS of preoperative axial neck pain was $30.7 \mathrm{~mm}(0-79)$ and 16.1 $\mathrm{mm}(0-80)$ in the C3-C6 and C3-C7 groups, respectively. Preoperative axial neck pain was not significantly different between both of the groups ( $p=0.1033)$. Postoperative axial neck pain at 1 year postoperatively was observed in seven of $11(63.6 \%)$ and nine of 22 patients (40.9\%) in the C3-C6 and C3-C7 groups, respectively. The mean VAS of postoperative axial neck pain was $21.4 \mathrm{~mm}(0-50)$ and $14.2 \mathrm{~mm}(0-80)$ in the C3-C6 and C3-C7 groups, respectively. Postoperative axial neck pain was not significantly different between both of the groups ( $p=0.2331$ ).

The mean preoperative JOA score was 11.0 (7.5-15.5) and 10.5 points (4-13), and the mean postoperative JOA score at 1 year postoperatively was $14.2(12.5-16.5)$ and 13.0 points (7-17) in the $\mathrm{C} 3-\mathrm{C} 6$ and $\mathrm{C} 3-\mathrm{C} 7$ groups, respectively. The mean JOA score improvement rate was $46.9 \%$ ( -66.7 to 83.3$)$ and $38.5 \%$ (-12.5 to 100$)$ in the C3$\mathrm{C} 6$ and $\mathrm{C} 3-\mathrm{C} 7$ groups, respectively. The pre- and postoperative JOA scores ( $p=0.8180, p=0.0947)$ and improvement rates of JOA score $(p=0.5161)$ were not significantly different between both of the groups.

\section{A representative case with severe $\mathrm{C} 8$ or $\mathrm{T} 1$ symptoms after C3-C6 laminoplasty}

\section{1) Case 1}

A 43-year-old man was diagnosed with CSM at C5-C6 by using a spinal cord evoked potential. He underwent C4C6 LP with C3 LN. Immediately after surgery, he com- plained of severe pain around the ulnar aspect of his left forearm, the T1 symptom (Fig. 4A). MRI showed that the spinal cord shifted posteriorly and impinged on the C7 lamina at 24 hours postoperatively. Two days postoperatively, he complained of numbness around the left lower extremity, which was suspected as a long tract sign. Two weeks postoperatively, the spinal cord shifted anteriorly and did not impinge on the C7 lamina; however, the highsignal change appeared in the spinal cord at $\mathrm{C} 6-\mathrm{C} 7$ on MRI (Fig. 4B). In this case, the distances of the posterior spinal cord shifting at C6 were $3.40 \mathrm{~mm}$ and $0.25 \mathrm{~mm}$ at 24 hours and 2 weeks postoperatively, respectively, and the distances of the posterior subarachnoid space at C7 were $2.00 \mathrm{~mm}$ preoperatively, and $0.00 \mathrm{~mm}$ and $1.12 \mathrm{~mm}$ at 24 hours and 2 weeks postoperatively, respectively. The T1 symptom was gradually relieved, and completely disappeared 6 months postoperatively.

\section{2) Case 2}

This was a case of a patient with severe $\mathrm{C} 8$ symptoms that our colleagues experienced. She was not included in our consecutive patients. A 72-year-old woman was diagnosed with CSM at C3-C4 by using a spinal cord evoked potential. She underwent C4-C6 LP with C3 LN. Immediately after surgery, she complained of severe pain around her left ring and little fingers, the $\mathrm{C} 8$ symptom. The next day, severe pain area extended to the ulnar aspect of her left forearm. MRI showed that the spinal cord shifted posteriorly and impinged on the $\mathrm{C} 7$ lamina at 24 hours postoperatively (Fig. 5). Two days postoperatively, she complained 


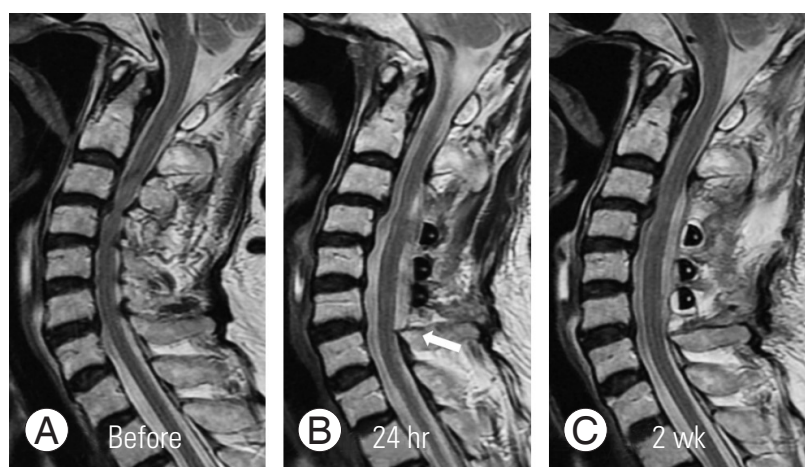

Fig. 5. Case 2 with severe C8 symptom. (A-C) The spinal cord shifted posteriorly and impinged on the $\mathrm{C} 7$ lamina on magnetic resonance imaging at 24 hours postoperatively. The spinal cord shifted anteriorly and did not impinge on the lamina 2 weeks postoperatively. Spinal cord or posterior fila radicularia would impinge on the lower end of the lamina (arrow).

of pain around her right ring and little fingers. Two weeks postoperatively, the spinal cord shifted anteriorly and did not impinge on the C7 lamina on MRI. In this case, the distances of the posterior spinal cord shifting at C6 were $3.63 \mathrm{~mm}$ at 24 hours and $3.16 \mathrm{~mm}$ at 2 weeks postoperatively, and the distances of the posterior subarachnoid space at C7 were $2.52 \mathrm{~mm}$ preoperatively, and $0.00 \mathrm{~mm}$ at 24 hours and $1.48 \mathrm{~mm}$ at 2 weeks postoperatively. The C8 symptom was gradually relieved and completely disappeared 20 days postoperatively.

\section{Discussion}

In this study, the incidence of postoperative $\mathrm{C} 8$ or $\mathrm{T} 1$ symptoms after C3-C6 LP was significantly higher than that after C3-C7 LP. Hosono et al. [8] reported that the diameter of the dural sac at C6/7 was significantly smaller in the C3-C6 LP, and the dural sac relatively compressed by the intact $\mathrm{C} 7$ lamina; however, they did not refer to $\mathrm{C} 8$ or T1 symptoms and any other complications. Because the posterior spinal cord shifting at C2-C6 was similar between both of the groups in the early postoperative period, the spinal cord would shift posteriorly and impinge on the C7 lamina after C3-C6 LP. In addition, the distance of the posterior subarachnoid space at $\mathrm{C} 7$ in the C3-C6 group was significantly shorter than that at $\mathrm{T} 1$ in the C3-C7 group at 24 hours postoperatively. Therefore, the posterior fila radicularia and spinal cord were more frequently impinged by the lower end of the lamina after LP in the C3-C6 group than in the C3-C7 group. We speculated that the difference in the distance of the pos- terior subarachnoid space at the lower end of the lamina after LP would cause the difference in the incidence of C8 or T1 symptoms between both of the groups.

C5 palsy was known as one of common complications after cervical LP. Several theories have been proposed to explain the occurrence of postoperative C5 palsy. Nerve root traction due to the spinal cord posterior shifting $[10,11]$, reperfusion spinal cord injury [12], surgical trauma [13], and the angle of elevated lamina [14] were proposed. We presumed that various factors caused C8 or T1 symptoms, such as C5 palsy. We proposed that the spinal cord or posterior fila radicularia impinged by the lower end of the lamina due to spinal cord posterior shifting would cause $\mathrm{C} 8$ or T1 symptoms. However, some cases with small distance of posterior subarachnoid space would have no $\mathrm{C} 8$ or T1 symptom (Fig. 3). The factors other than the impingement between the spinal cord or posterior fila radicularia and lower end of the lamina would relate to $\mathrm{C} 8$ or $\mathrm{T} 1$ symptoms.

These new C8 or T1 symptoms spontaneously resolved 2-3 weeks postoperatively. Shiozaki et al. [10], prospectively examining the posterior shift of the spinal cord on MRI in 19 consecutive patients at 24 hours and 2 weeks after LP, reported that the posterior shift of the spinal cord at 24 hours tend to shift more posteriorly than that at 2 weeks after LP. Similarly, postoperative spinal cord posterior shifting at 24 hours postoperatively was greater than that at 2 weeks postoperatively. We speculated that the impingement of the dura sac or the cervical spinal cord on the lamina was released, and C8 or T1 symptoms would be cured.

Postoperative axial neck pain is a noticeable problem associated with cervical LP. In this study, the VAS scores of pre- and postoperative axial neck pain were not significantly different between both of the groups. Some studies have reported the relationship between the preservation of nuchal muscles and the clinical outcome, particularly postoperative axial neck pain in LP. Sakaura et al. [5] reported that the frequencies of persistent axial neck pain and loss of cervical lordosis after LP decreased significantly for 8 to 10 years in 31 patients after C3-C6 LP. In contrast, Takeuchi et al. [6] changed the laminoplastic procedure from LP with reattachment of the SSC at $\mathrm{C} 2$ to LP with C3 LN, preserving the SSC at C2; the modified LP demonstrated that the preserved the SSC significantly reduced postoperative axial neck pain and maintained the entire cervical posterior muscular volume on MRI 
compared with conventional LP. Kowatari et al. [15] reported no significant differences in incidence, intensity, or severity of axial symptoms at 1 year postoperatively between C3-C6 LP and C3-C7 LP. Anatomical studies on posterior cervical muscular were reported. Ono et al. [7], examining the distribution of posterior cervical muscles by using 50 cadavers, reported that preserving $\mathrm{C} 7$ spinous process in C3-C6 LP reduced invasion of the nuchal muscles. In this study, because both the SSC in C2 and ligament in $\mathrm{C} 7$ were preserved in both of the groups, the postoperative axial neck pain was not significantly different between both of the groups. The clinical results in the postoperative JOA score and improvement rate of JOA score were not significantly different between both of the groups, which was similar to previous reports $[5,8]$.

\section{Conclusions}

The incidence of C8 or T1 symptoms in C3-C6 LP was significantly higher than that in C3-C7 LP. The spinal cord would shift posteriorly and impinge on the intact lamina postoperatively in the early postoperative period. The posterior fila radicularia and the spinal cord impinged on the lower end of the lamina would be one of causes of C8 or T1 symptoms. Clinical outcomes in the axial neck pain, JOA score, and JOA score improvement rate did not differ significantly between both of the groups.

\section{Conflict of Interest}

No potential conflict of interest relevant to this article was reported.

\section{ORCID}

Hitoshi Kudo: https://orcid.org/0000-0003-2118-1499 Kazunari Takeuchi: https://orcid.org/0000-0002-5480-3744 Toru Yokoyama: https://orcid.org/0000-0002-9062-8701 Yoshihito Yamasaki: https://orcid.org/0000-0002-7973-3367 Kanichiro Wada: https://orcid.org/0000-0002-1112-5656 Gentaro Kumagai: https://orcid.org/0000-0003-0597-6603 Toru Asari: https://orcid.org/0000-0002-4767-8519 Hironori Otsuka: https://orcid.org/0000-0001-7896-0819 Yasuyuki Ishibashi: https://orcid.org/0000-0003-2180-7922

\section{References}

1. Seichi A, Takeshita K, Ohishi I, et al. Long-term results of double-door laminoplasty for cervical stenotic myelopathy. Spine (Phila Pa 1976) 2001;26:47987.

2. Nakano K, Harata S, Suetsuna F, Araki T, Itoh J. Spinous process-splitting laminoplasty using hydroxyapatite spinous process spacer. Spine (Phila $\mathrm{Pa} 1976$ ) 1992;17:S41-3.

3. Sakaura H, Hosono N, Mukai Y, Ishii T, Yoshikawa H. C5 palsy after decompression surgery for cervical myelopathy: review of the literature. Spine (Phila Pa 1976) 2003;28:2447-51.

4. Takeuchi K, Yokoyama T, Ono A, et al. Limitation of activities of daily living accompanying reduced neck mobility after laminoplasty preserving or reattaching the semispinalis cervicis into axis. Eur Spine J 2008;17:415-20.

5. Sakaura H, Hosono N, Mukai Y, Iwasaki M, Yoshikawa H. C3-6 laminoplasty for cervical spondylotic myelopathy maintains satisfactory long-term surgical outcomes. Global Spine J 2014;4:169-74.

6. Takeuchi K, Yokoyama T, Aburakawa S, et al. Axial symptoms after cervical laminoplasty with C3 laminectomy compared with conventional C3-C7 laminoplasty: a modified laminoplasty preserving the semispinalis cervicis inserted into axis. Spine (Phila Pa 1976) 2005;30:2544-9.

7. Ono A, Tonosaki Y, Numasawa T, et al. The relationship between the anatomy of the nuchal ligament and postoperative axial pain after cervical laminoplasty: cadaver and clinical study. Spine (Phila $\mathrm{Pa} 1976$ ) 2012;37:E1607-13.

8. Hosono N, Sakaura H, Mukai Y, Fujii R, Yoshikawa H. C3-6 laminoplasty takes over C3-7 laminoplasty with significantly lower incidence of axial neck pain. Eur Spine J 2006;15:1375-9.

9. Takeuchi K, Yokoyama T, Aburakawa S, et al. Postoperative changes at the lower end of cervical laminoplasty: for preservation of the $\mathrm{C} 7$ spinous process in laminoplasty. J Spinal Disord Tech 2006;19:402-6.

10. Shiozaki T, Otsuka H, Nakata Y, et al. Spinal cord shift on magnetic resonance imaging at 24 hours after cervical laminoplasty. Spine (Phila Pa 1976) 2009;34:274-9.

11. Tsuzuki N, Tanaka H, Abe R, Horita Y, Okai K. Cer- 
vical radiculopathy occurring after the posterior decompression of cervical spinal cord. Rinsho Seikei Geka 1991;26:525-34.

12. Chiba K, Toyama Y, Matsumoto M, Maruiwa H, Watanabe M, Hirabayashi K. Segmental motor paralysis after expansive open-door laminoplasty. Spine (Phila Pa 1976) 2002;27:2108-15.

13. Hirabayashi K, Satomi K, Ichimura S, Tanaka K, Wakano K, Toyama Y. Complication of expansive open-door laminoplasty for ossification of the poste- rior longitudinal ligament in cervical spine. Rinsho Seikei Geka 1988;23:509-15.

14. Uematsu Y, Tokuhashi Y, Matsuzaki H. Radiculopathy after laminoplasty of the cervical spine. Spine (Phila Pa 1976) 1998;23:2057-62.

15. Kowatari K, Ueyama K, Sannohe A, Yamasaki Y. Preserving the $\mathrm{C} 7$ spinous process with its muscles attached: effect on axial symptoms after cervical laminoplasty. J Orthop Sci 2009;14:279-84. 